\title{
Gleichschenklige Teildreiecke im regelmäßigen Vieleck
}

\author{
Dietrich Francke \\ Dietrich Francke hat an der TU Dresden ein Diplom-Ingenieurstudium in Elektrotech- \\ nik absolviert und war später als Leiter einer Entwicklungsabteilung für elektronische \\ Spezialmeßgeräte tätig.
}

\section{Einleitung}

Die im folgenden beschriebene elementare Eigenschaft der Diagonalendreiecke im regelmäßigen Vieleck wurde im Jahr 2005 bei Untersuchungen der $n$ komplexen Einheitswurzeln $z_{k}$ der Kreisteilungsgleichung $z^{n}-1=0$ und der goniometrischen und algebraischen Bestimmungsgleichungen für regelmäßige Vielecke gefunden. Weitgehende Recherchen hierzu erbrachten nur wenige spezielle Literaturstellen $[1$, S. 203], [2, S. 63, Fig. 4.6c, (4.6. Regular, impossible tesselations)].

Regelmäßige Vielecke mit ungerader Eckenzahl $n$ bzw. mit gerader Eckenzahl $n^{\prime}$ seien in Diagonalendreiecke zerlegt, die von je zwei der von derselben Ecke des Vielecks ausgehenden Haupt- bzw. Nebendiagonalen und jeweils einer Seite $a$ des regelmäßigen Vielecks gebildet sind und deren Spitzen daher auf dieser Ecke des Vielecks liegen. Nach dem Peripheriewinkelsatz sind ihre Spitzenwinkel $\alpha=\pi / n$ bzw. $\alpha=\pi / n^{\prime}$ gleich groß.

Diese Haupt- bzw. Nebendiagonalendreiecke lassen sich ihrerseits in jeweils eine Aneinanderreihung von gleichschenkligen Teildreiecken mit der Schenkellänge $a$ zerlegen. Dieser Sachverhalt ermöglicht es, das bzw. die Hauptdiagonalendreiecke darzustellen und

Ein Hauptdiagonalendreieck $A B C$ in einem regelmäßigen Vieleck ungerader Eckenzahl wird von den beiden gleichlangen Hauptdiagonalen $A B$ und $A C$, die von der Ecke $A$ ausgehen, und von der $A$ gegenüberliegenden Seite $B C=a$ des Vielecks gebildet. In dem nachfolgenden Beitrag zeigt der Autor, wie sich das Hauptdiagonalendreieck ausgehend von der Spitze $A$ vollständig in aneinandergereihte gleichschenklige Dreiecke zerlegen lässt, deren Schenkel gleichlang wie die Seite $a$ sind. Dieser geometrische Sachverhalt lässt sich durch ein stabiles Stabwerk modellieren, das aus einem Stellwinkel und untereinander gelenkig verbundenen Stücken gleicher Länge $a$ besteht, die zusätzlich auf dessen Schenkeln parallelverschiebbar gelagert sind. 
weiter von diesen ausgehend das ganze regelmäßige Vieleck elementar, also mittels Zirkel und Lineal zu konstruieren.

Nach den Gaußschen Kriterien läßt sich nur eine kleine Auswahl regelmäßiger Vielecke ausschließlich mit Zirkel und Lineal konstruieren, während bei den folgenden Betrachtungen für die Eckenzahlen $n$ bzw. $n^{\prime}$ der regelmäßigen Vielecke keine Einschränkungen gelten sollen.

\section{Das Hauptdiagonalendreieck im regelmäßigen Vieleck mit ungerader Eckenzahl $n$}

Das Hauptdiagonalendreieck $A B C$ in einem regelmäßigen Vieleck mit einer ungeraden Eckenzahl $n$ wird von den beiden gleichlangen Hauptdiagonalen $A B$ und $A C$, die von einer Ecke $A$ des Vielecks ausgehen, und von der dieser Ecke $A$ gegenüberliegenden Seite $B C=a$ des regelmäßigen Vielecks gebildet (Abb. 1).

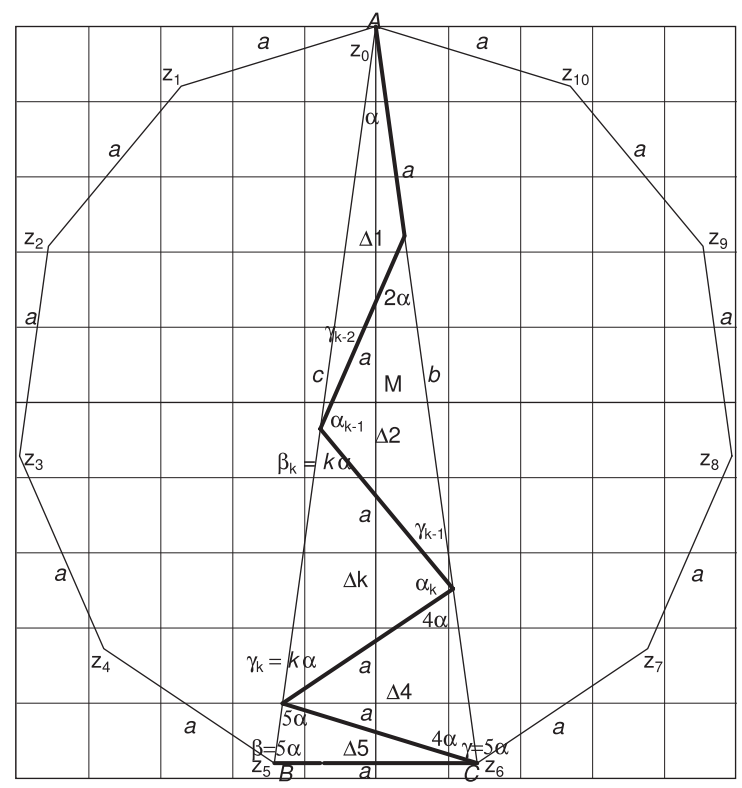

Abb. 1 Gleichschenkliges Dreieck mit ganzzahligem Basis-/Spitzenwinkel-Verhältnis $m=5$ und der Basis $a$, zugleich das Hauptdiagonalendreieck $A B C$ über einer Seite $a$ eines regelmäßigen Vielecks mit ungerader Eckenzahl $n=2 m+1=11$, zerlegt in $m$ Teildreiecke $\Delta 1$ bis $\Delta m$ mit gleicher Schenkellänge $a, \alpha=\pi / n=\pi / 11, \beta=\gamma=m \alpha=5 \alpha$.

Da das Hauptdiagonalendreieck $A B C$ demnach gleichschenklig ist, sind die Basiswinkel $\beta$ und $\gamma$ gleich groß.

Wie man leicht errechnen kann, führt die Winkelsumme in dem gleichschenkligen Hauptdiagonalendreieck $\alpha+2 \beta=\alpha+2 \gamma=\pi$ mit dem Spitzenwinkel $\alpha=\pi / n$ zu dem 
Basis-Spitzenwinkel-Verhältnis

$$
m=\beta / \alpha=\gamma / \alpha=(n-1) / 2 .
$$

Wegen der Ungeradzahligkeit der Eckenzahl $n$ ist der Ausdruck $(n-1) / 2$ ganzzahlig, d.h., die Basiswinkel $\beta$ und $\gamma$ sind ganzzahlige Vielfache $m=(n-1) / 2$ des Spitzenwinkels $\alpha$. Wendet man den Außenwinkelsatz auf die Folge der gleichschenkligen Teildreiecke $\triangle 1$ bis $\triangle m$ an (s. Abb. 1), so beträgt der Außenwinkel $\alpha_{1}$ des 1 . Teildreiecks $2 \cdot \alpha$, der zugleich der Basiswinkel $\beta_{2}$ des 2 . Teildreiecks ist, usw. Durch vollständige Induktion läßt sich zeigen, daß der Basiswinkel $\beta_{k}$ des $k$-ten Teildreiecks $\triangle k$ gleich $2 \cdot(k-1) \alpha-(k-2) \alpha=k \alpha$ und somit der Basiswinkel $\beta_{m}$ des letzten Teildreiecks $\triangle m$ gleich $m \alpha$ ist.

Der Basiswinkel $\beta_{m}=m \alpha$ des $m$-ten Teildreiecks $\triangle m$ stimmt nach (1) mit dem Basiswinkel $\beta=m \alpha$ bzw. $\gamma=m \alpha$ des ganzen Hauptdiagonalendreiecks $A B C$ überein, womit nachgewiesen ist, daß sich das Hauptdiagonalendreieck in einem regelmäßigen Vieleck mit ungerader Eckenzahl $n$ vollständig in $m$ aneinandergereihte gleichschenklige Teildreiecke $\Delta k, k=1, \ldots, m$, zerlegen läßt, deren Schenkel gleich lang wie die Basis $a$ des Hauptdiagonalendreiecks und damit gleich lang wie eine Seite $a$ des regelmäßigen Vielecks sind.

\section{Das Hauptdiagonalendreieck im regelmäßigen Vieleck mit gerader Eckenzahl $\boldsymbol{n}^{\prime}$}

Das Hauptdiagonalendreieck $A B C$ in einem regelmäßigen Vieleck mit einer geraden Eckenzahl $n^{\prime}$ wird von der Hauptdiagonalen $A B$ und einer der beiden benachbarten gleichlangen Nebendiagonalen $A C$, die von einer Ecke $A$ des Vielecks ausgehen, und von der dieser Ecke $A$ gegenüberliegenden Seite $B C=a$ des regelmäßigen Vielecks gebildet (Abb. 2).

Da die Hauptdiagonale $A B$ ein Durchmesser des Umkreises des regelmäßigen Vielecks ist, ist nach dem Satz des Thales das Hauptdiagonalendreieck $A B C$ rechtwinklig, d.h. $\gamma=\pi / 2$.

Wie leicht zu errechnen ist, ist der Spitzenwinkel $\alpha=\pi / n^{\prime}$ und somit das KathetenHypotenusenwinkel-Verhältnis

$$
m=\beta / \alpha=n^{\prime} / 2-1 .
$$

Wegen der Geradzahligkeit der Eckenzahl $n^{\prime}$ ist der Ausdruck $n^{\prime} / 2-1$ ganzzahlig, d.h., der Katheten-Hypotenusenwinkel $\beta$ ist ein ganzzahliges Vielfaches $m=n^{\prime} / 2-1$ des Spitzenwinkels $\alpha$.

Es soll hergeleitet werden, daß auch im regelmäßigen Vieleck mit gerader Eckenzahl $n^{\prime}$ jedes Hauptdiagonalendreieck $A B C$ vollständig in $m$ aneinandergereihte gleichschenklige Teildreiecke $\triangle 1$ bis $\triangle m$ mit einer einheitlichen Schenkellänge $a$ zerlegt werden kann, die der Kathete $a$ des ganzen Hauptdiagonalendreiecks $A B C$ und damit zugleich einer Seite $a$ des regelmäßigen Vielecks entspricht.

Wendet man den Außenwinkelsatz auf die Folge der gleichschenkligen Teildreiecke $\triangle 1$ bis $\triangle m$ an, so ist der Basiswinkel $\beta_{k}$ des $k$-ten Teildreiecks $\triangle k$ gleich $k \alpha$ und der Basiswinkel $\beta_{m}$ des letzten Teildreiecks $\triangle m$ gleich $m \alpha$. 


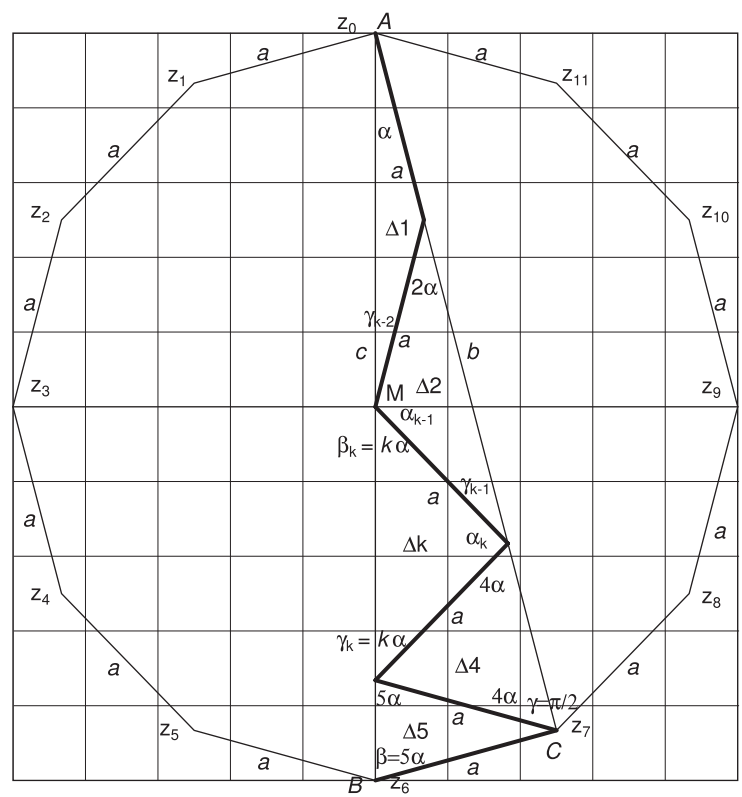

Abb. 2 Rechtwinkliges Dreieck mit ganzzahligem Katheten-HypotenusenwinkelVerhältnis $m=5$ und der Basis $a$, zugleich ein Hauptdiagonalendreieck $A B C$ über einer Seite $a$ eines regelmäßigen Vielecks mit gerader Eckenzahl $n^{\prime}=2(m+1)=12$, zerlegt in $m$ Teildreiecke $\Delta 1$ bis $\triangle m$ mit gleicher Schenkellänge $a, \alpha=\pi / n^{\prime}=\pi / 12, \beta=m \alpha=5 \alpha, \gamma=\pi / 2$.

Der Basiswinkel $\beta_{m}=m \alpha$ des $m$-ten Teildreiecks $\triangle m$ stimmt nach (2) mit dem KathetenHypotenusenwinkel $\beta=m \alpha$ des ganzen Hauptdiagonalendreiecks $A B C$ überein, womit nachgewiesen ist, daß sich ein Hauptdiagonalendreieck in einem regelmäßigen Vieleck mit gerader Eckenzahl $n^{\prime}$ vollständig in $m$ aneinandergereihte gleichschenklige Teildreiecke $\Delta k, k=1, \ldots, m$, zerlegen läßt, deren Schenkel gleich lang wie die Kathete $a$ des Hauptdiagonalendreiecks und damit gleich lang wie eine Seite $a$ des regelmäßigen Vielecks sind.

\section{Die konstruktive Darstellung eines Hauptdiagonalendreiecks für ein regelmäßiges Vieleck mit ungerader Eckenzahl $\boldsymbol{n}$}

Das Ergebnis von Abschnitt 1, demzufolge ein Hauptdiagonalendreieck in eine bestimmte Anzahl von gleichschenkligen Teildreiecken mit gegebener Schenkellänge $a$ zerlegt werden kann, soll daher zu einer konstruktiven Darstellung benutzt werden.

Das Hauptdiagonalendreieck $A B C$ für ein regelmäßiges Vieleck mit ungerader Eckenzahl $n$, das wegen seiner Gleichschenkligkeit symmetrisch ist, soll in zwei zur Symmetrieachse spiegelbildliche Aneinanderreihungen von je $m$ gleichschenkligen Teildreiecken mit der Schenkellänge $a$ zerlegt werden (Abb. 3). 


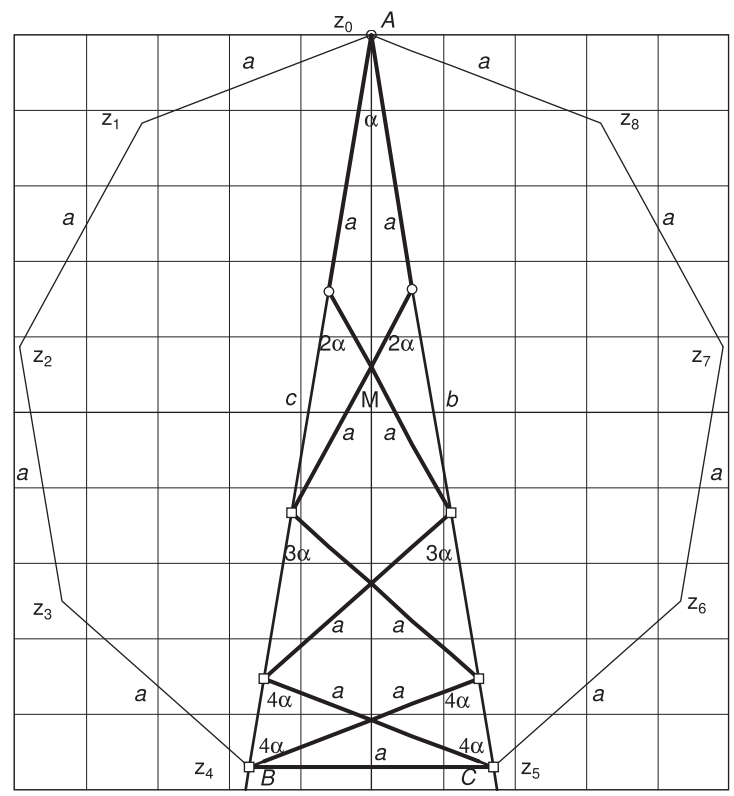

Abb. 3 Darstellung des Hauptdiagonalendreiecks $A B C$ für ein regelmäßiges Vieleck mit der ungeraden Eckenzahl $n=9$ als Stabwerk aus den im Eckpunkt $A$ drehbar verbundenen Schenkeln $b$ und $c$ eines Stellwinkels $\alpha$ und aus $n=9$ gelenkig und parallelverschiebbar verbundenen Distanzstücken von der gleichen Länge $a$. $\bigcirc$ und $\square=$ Gelenkpunkte zwischen den Distanzstücken gleicher Länge $a . \square=$ außerdem auf den Schenkeln $b$ und $c$ parallelgeführte Gelenkpunkte $\bigcirc$.

Da benachbarte Teildreiecke jeweils einen gemeinsamen Schenkel der Länge $a$ besitzen, ist die Gesamtzahl dieser Teildreiecksschenkel gleich der Anzahl der Teildreiecke 2m, zuzüglich eines gemeinsamen Schenkels der $m$-ten Teildreiecke, also gleich $2 m+1$; sie ist damit gleich der Eckenzahl $n=2 m+1$. Die $n$ Seiten des ganzen regelmäßigen Vielecks entstehen durch Parallelverschiebung dieser $n$ Teildreiecksschenkel.

In Abb. 4 ist das Funktionsmodell eines Stabwerkes für das zu einem regelmäßigen Vieleck mit der ungeraden Eckenzahl $n=11$ gehörende Hauptdiagonalendreieck dargestellt.

\section{Die konstruktive Darstellung von Hauptdiagonalendreiecken für ein regelmäßiges Vieleck mit gerader Eckenzahl $\boldsymbol{n}^{\prime}$}

Das Ergebnis von Abschnitt 2, demzufolge ein Hauptdiagonalendreieck in eine bestimmte Anzahl von gleichschenkligen Teildreiecken mit gegebener Schenkellänge $a$ zerlegt werden kann, soll daher zu einer konstruktiven Darstellung benutzt werden.

Das Hauptdiagonalendreieck $A B C$ für ein regelmäßiges Vieleck mit gerader Eckenzahl $n^{\prime}$, das wegen seiner Rechtwinkligkeit unsymmetrisch ist, wird durch das spiegelbildliche und daher ebenfalls rechtwinklige Dreieck $A B^{\prime} C$ zu einem gleichschenkligen und daher symmetrischen Dreieck $A B B^{\prime}$ ergänzt, dessen Basis die Länge $2 a$ hat. Ähnliche 


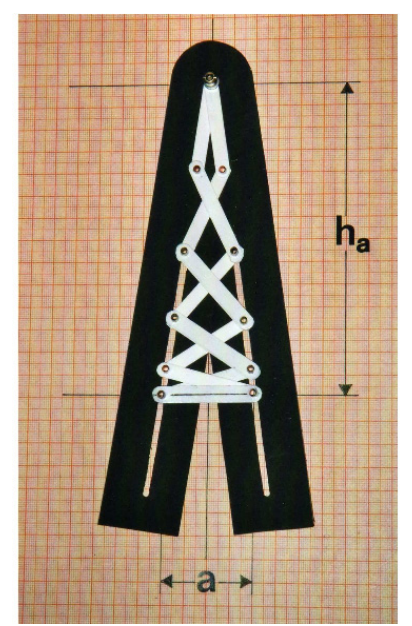

Abb. 4 Funktionsmodell eines Stabwerkes für das zu einem regelmäßigen Vieleck mit der ungeraden Eckenzahl $n=11$ gehörende Hauptdiagonalendreieck, bestehend aus einem gleichschenkligen Stellwinkel $\alpha$ und $n$ mit dessen Schenkeln und untereinander gelenkig verbundenen und zusätzlich parallelverschiebbar gelagerten Distanzstücken der Länge $a$.

Überlegungen wie in Abschnitt 3 führen nun zu Zerlegungen dieser beiden Hauptdiagonalendreiecke $A B C$ und $A B^{\prime} C$ in zwei spiegelbildliche Aneinanderreihungen von je $m$ gleichschenkligen Teildreiecken mit der Schenkellänge $a$ (Abb. 5).

Da benachbarte Teildreiecke jeweils einen gemeinsamen Schenkel der Länge $a$ besitzen, ist die Gesamtzahl dieser Teildreiecksschenkel gleich der Anzahl der Teildreiecke $2 m+2$ bei geradzahligem $m$ bzw. $2 m+1$ bei ungeradzahligem $m$, wegen des dabei entstehenden gemeinsamen Schenkels der ersten beiden Teildreiecke. Die $n^{\prime}$ Seiten des ganzen regelmäßigen Vielecks entstehen durch Parallelverschiebung dieser $n^{\prime}$ bzw. $n^{\prime}-1$ Teildreiecksschenkel.

\section{Die Zerlegbarkeit eines regelmäßigen Vielecks in ein Netz aus Rhomben gleicher Seitenlänge $a$}

In ein regelmäßiges Vieleck mit einer ungeraden oder geraden Eckenzahl $n$ bzw. $n^{\prime}$ seien von einer Ecke $A$ aus alle Haupt- und Nebendiagonalen eingetragen, so daß das Vieleck in $n-2$ bzw. $n^{\prime}-2$ Diagonalendreiecke mit dem gleichen Spitzenwinkel $\alpha=\pi / n$ bzw. $\alpha=\pi / n^{\prime}$ zerlegt ist, die aus je zwei benachbarten Diagonalen und einer Seite $a$ des Vielecks bestehen.

Aus Abb. 6 bzw. Abb. 7 ist zu ersehen, daß sich ein regelmäßiges Vieleck vollständig in Teildreiecke $\triangle k i, k=1, \ldots, m-i, i=0, \ldots, m-1$, zerlegen läßt und die spiegelbildlichen Teildreiecke $\Delta k$ sich paarweise zu Rhomben mit der Seitenlänge $a$ ergänzen lassen, so daß das Vieleck, ausgehend von seinem gleichschenkligen Hauptdiagonalendreieck $A B C$ bzw. von seinen rechtwinkligen Hauptdiagonalendreiecken $A B C$ und $A B C^{\prime}$, sich auch in ein Netz aus zusammenhängenden Rhomben zerlegen läßt (Abb. 8). 


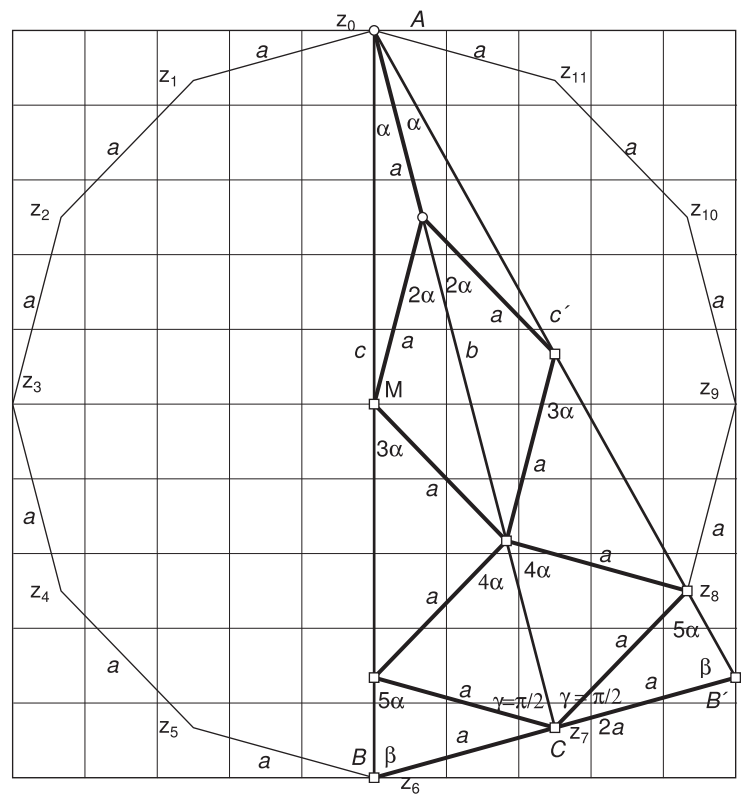

Abb. 5 Darstellung der spiegelbildlichen Hauptdiagonalendreiecke $A B C$ und $A B^{\prime} C$ für ein regelmäßiges Vieleck mit der geraden Eckenzahl $n^{\prime}=12$ als Stabwerk aus den im Eckpunkt $A$ drehbar verbundenen Schenkeln $b, c$ und $c^{\prime}$ eines Stellwinkels $2 \alpha$ und aus 9 gelenkig und parallelgeführt verbundenen Stäben der Länge $a$ und einem Stab der Länge $2 a$. $\bigcirc$ und $\square=$ Gelenkpunkte zwischen den Distanzstücken der Längen $a$ und $2 a$. $\square=$ außerdem auf den Schenkeln $b, c$ und $c^{\prime}$ parallelgeführte Gelenkpunkte $\bigcirc$.

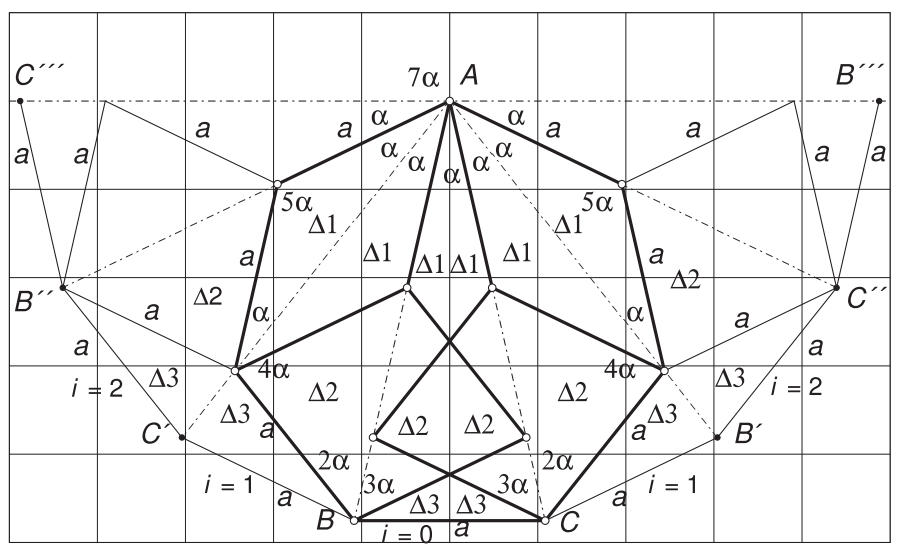

Abb. 6 Zerlegung eines regelmäßigen Vielecks mit der ungeraden Eckenzahl $n=7$, $m=3$, und der Seitenlänge $a$ in $n-2$ Diagonalendreiecke $i=0, \ldots, m-1$, und diese wiederum in gleichschenklige Teildreiecke $\Delta k, k=1, \ldots, m-i$, die ein Netz aus zusammenhängenden Rhomben von der gleichen Seitenlänge $a$ des Vielecks bilden. 


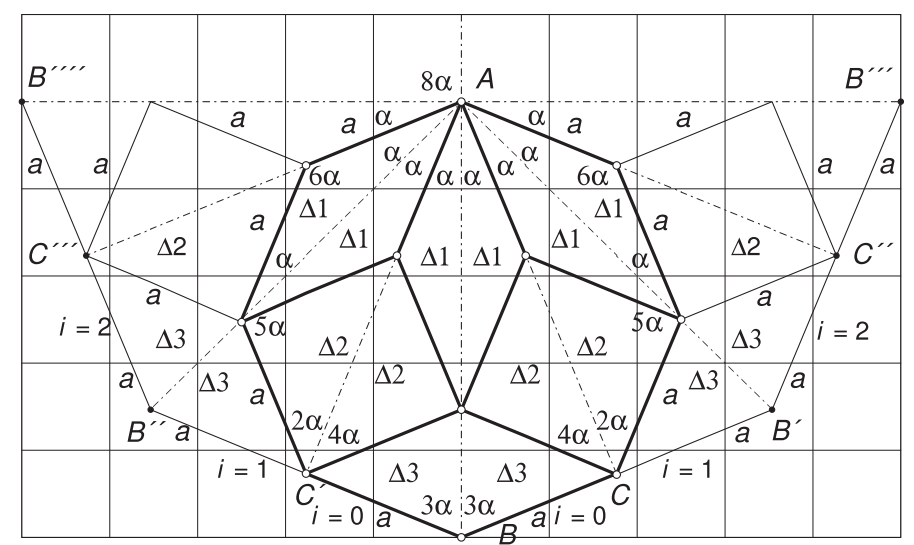

Abb. 7 Zerlegung eines regelmäßigen Vielecks mit der geraden Eckenzahl $n^{\prime}=8$, $m=3$, und der Seitenlänge $a$ in $n^{\prime}-2$ Diagonalendreiecke $i=0, \ldots, m-1$, und diese wiederum in gleichschenklige Teildreiecke $\Delta k, k=1, \ldots, m-i$, die ein Netz aus zusammenhängenden Rhomben von der gleichen Seitenlänge $a$ des Vielecks bilden.

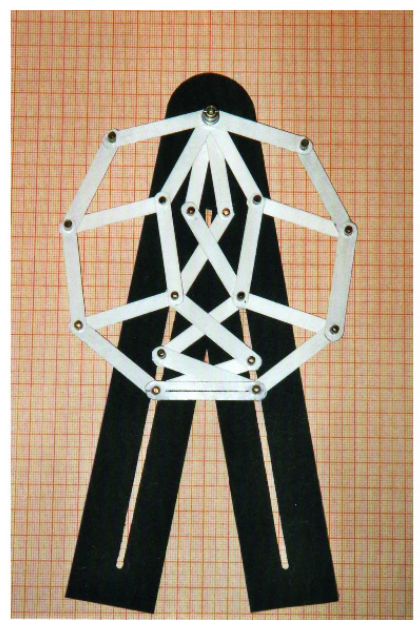

Abb. 8 Funktionsmodell eines Stabwerkes für ein regelmäßiges Vieleck mit der ungeraden Eckenzahl $n=9$ als ein Netz aus zusammenhängenden Rhomben gleicher Seitenlänge $a$, wobei die beiden Hauptdiagonalen zur eindeutigen Bestimmung der Regelmäßigkeit des Vielecks durch Parallelführung der zugehörigen Gelenkpunkte notwendig sind. 
Dabei liegen gleichliegende Eckpunkte der Rhomben mit gleichem Spitzenwinkel $k$. $2 \alpha, k=1, \ldots, m$, auf Halbkreisen um die gemeinsame Spitze $A$ der Diagonalendreiecke. Aus Abb. 8 ist auch zu entnehmen, daß ein nur in Rhomben zerlegtes regelmäßiges Vieleck ohne Zuhilfenahme des bzw. der Hauptdiagonalendreiecke ein instabiles Stabwerk darstellen würde, d.h., es ließe sich zu einem beliebigen unregelmäßigen Vieleck umgestalten.

\section{Literatur}

[1] Gericke, H.: Mathematik in Antike, Orient und Abendland. Teil I, Matrixverlag, Wiesbaden 2005.

[2] Coxeter, H.S.M.: Introduction to Geometry. Wiley Classics Library, 2. Edition 1989.

Dietrich Francke

Salvador-Allende-Str. 13

D-12559 Berlin, Deutschland

e-mail: dietrich-francke@gmx.de 